\title{
Online MOS Capacitor Characterization in LabVIEW Environment
}

\author{
$\underline{\text { doi:10.3991/ijoe.v5s1.1000 }}$ \\ S. C. Pandey, A. Maiti ${ }^{1}$, T. K. Maiti and C. K. Maiti \\ Indian Institute of Technology, Kharagpur, India
}

\begin{abstract}
We present an automated evaluation procedure to characterize MOS capacitors involving high-k gate dielectrics. Suitability of LabVIEW environment for online web-based semiconductor device characterization is demonstrated. Developed algorithms have been successfully applied to automate the MOS capacitor measurements for Capacitance-Voltage, Conductance-Voltage and CurrentVoltage characteristics. Implementation of the algorithm for use as a remote internet-based characterization tool where the client and server communicate with each other via web services is also shown.
\end{abstract}

Index Terms-LabVIEW, MOS capacitor characterization, virtual instrumentation

\section{INTRODUCTION}

The availability of the Internet as a communication medium has opened up a wide range of possibilities for extending its use into new areas. User-friendly, computercontrolled instrumentation and data analysis techniques are revolutionizing the measurement techniques, allowing automated measurements online. The remote, web-based experimentation augments the laboratory experience of the students by offering access to sophisticated instrumentation. It provides a natural and important extension of the traditional laboratory component, which normally uses relatively simple equipment.

The International Technology Roadmaps for Semiconductors (ITRS) identifies a number of challenges for continued successful scaling of MOSFET technology. It is clear that new materials and process modules will be needed to meet the ITRS roadmap requirements and enhance performance for a given technology node. Lowpower applications such as battery operated handheld devices require a reduced gate leakage current. To reduce the gate leakage, standard oxynitride gate insulators will be replaced by high-k dielectrics. Among the promising candidates for the $45 \mathrm{~nm}$ technology node applications are $\mathrm{Y}_{2} \mathrm{O}_{3}$ and $\mathrm{Ta}_{2} \mathrm{O}_{5}$ with a high-k value in the range 15-30. The main issues related to these types of dielectric materials, which still have to be addressed by researchers, are the high number of fixed/trapped charges and interface states. Both threshold voltage stability and low-field mobility are adversely affected by the high amount of charge present in the high-k dielectrics.

High-k materials are often used in combination with different metal gate electrodes and results in increased $D_{\text {it }}$ and the number of fixed charges which also influence the low-frequency noise. Thus the study of fixed/trapped charges and interface states in high-k dielectrics is impor- tant. However, the measurements involve huge amount of data collection which demands automated testing. Ideally, a large volume of devices should be tested so that average figures and distributions may be determined. In accomplishing the collection and processing of such a large quantity of data, manual handling becomes a serious limitation. In most cases, the data obtained from various sources (instruments) must be directed to a computer for analysis. Often one finds several generations of apparatus in a laboratory, each with its own post-processing system or software. It is advantageous to tie all of these separate systems together so that all operations can share a common platform and analysis can span relevant measurements without manual re-entry, disk swapping, or data conversion.

Thus a bottleneck in the flow of measurement results is eliminated. One promising system for the integration and automation of laboratory equipment is LabVIEW from National Instruments. In this article, we present an automatic measurement bench programmable by the IEEE-488 bus that permits automated characterization MOS capacitors. The characterization involves extraction of Capacitance-Voltage (C-V), Conductance-Voltage (G-V) and Leakage Current Density Voltage $(\mathrm{J}-\mathrm{V})$ characteristics. The measurement bench is managed by a program, written by a graphical language LabVIEW from National Instruments.

\section{LABVIEW ENVIRONMENT}

LabVIEW is a program development application, much like a $\mathrm{C}, \mathrm{C}++$ or BASIC software development tool. It is, however, different from those applications in one important aspect. LabVIEW uses a graphical language $(\mathrm{G})$ to create programs in block diagram form, while other programming tools use text-based languages. LabVIEW includes libraries of functions and development tools designed specifically for instrument control. It has application specific libraries for data acquisition, serial instrument control, data analysis, data presentation and data storage [LabVIEW 8.507]. The LabVIEW system provides an intuitive, graphical interface for the automated control of laboratory equipment. For each specific type of measurement, a virtual instrument (VI) is created. Virtual instrument is a programming technique and use a general purpose computer to mimic real instruments with their dedicated controls and displays - but with the added versatility that comes with the software.

The VI can be implemented in normal programming languages like $\mathrm{C}, \mathrm{C}++$, Pascal, VB, VC etc., but the advent of graphic languages (' $G$ ' languages) like LabVIEW, 


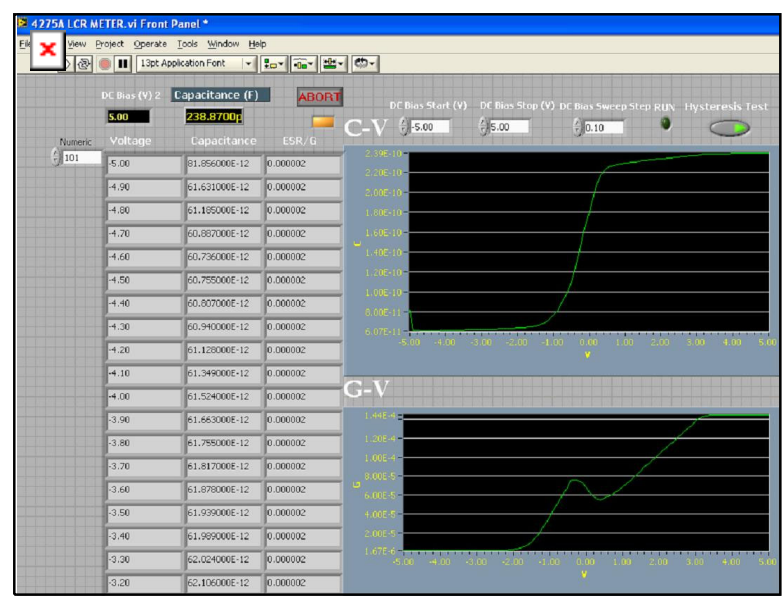

Figure 1(a): Graphical user interface of the program written in LabVIEW for measurement of C-V and G-V.

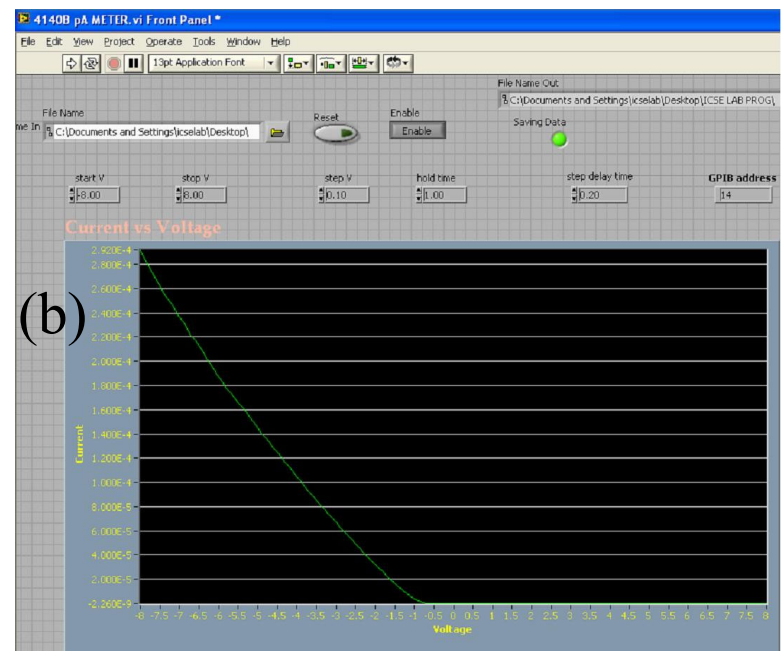

Figure 1(b): Graphical user interface of the program written in LabVIEW for measurement of I-V.

HP-VEE, Test point, Agilent VIEW etc., has made the VI implementation easier. VI implementation is cost effective, flexible and customizable than other tools. All of the measurement parameters can be varied by manipulation from the front panel as shown in Figures 1(a) and (b) which are the graphical equivalent of the push buttons and knobs on a test set, allowing a user who is not familiar with the programming to operate the setup easily.

On the front panel of a VI, the controls and data displays for our system are placed by selecting objects from the Controls palette, such as numeric displays, knob, meters, gauges, thermometers, Array, Matrix, Cluster, charts and graphs. When we complete and run the VI, we use the front panel to control our system whether we move a slide, zoom in on a graph, or enter a value with the keyboard. The programming which coordinates the operation is accomplished graphically using a dataflow language. Actual program included in the block diagram of the panel window is shown in Figures 2(a) and (b).

To program the VI, we constructed the block diagram based on the detail of text-based programming languages. This is done by selecting objects (icons) from the Functions palette and connecting them together with wires to transfer data among block diagram objects. These objects include simple arithmetic functions, advanced acquisition and analysis routines, network and file $\mathrm{I} / \mathrm{O}$ operations, and more [NI/LabVIEW 8.507]. An example the graphical language is presented in Figures 2(a) and 2(b). Since the whole system is based on a PC, the data is easily ported in ASCII form to other programs or even shared via server with other PCs. In Figures 1(a) and (b), input parameters such as frequency, process parameters are the signal level. Capacitor $(\mathrm{C})$, conductance $(\mathrm{G})$ and leakage current density $(\mathrm{J})$ which are extracted from $\mathrm{C}-\mathrm{V}, \mathrm{C}-\mathrm{G}$ and $\mathrm{I}-\mathrm{V}$ curve is specified in front panel. The output parameters are capacitor $(\mathrm{C})$, conductance $(\mathrm{G})$ and leakage current density (J).

\section{RESULT AND DiscUSSIONS}

The flow chart developed by us and used for $\mathrm{C}-\mathrm{V}, \mathrm{G}-\mathrm{V}$, and $\mathrm{I}-\mathrm{V}$ measurements is shown in Figure 3. In this section a brief introduction to the HP4275A LCR meter and HP4140B pA meter is given. Subsequently, setting up of the HP4275A LCR meter and HP4140B pA meter for measurement of capacitance, conductance and leakage current density is explained. The HP4275A LCR meter and HP4140B pA meter is general purpose meter used to measure capacitor $(\mathrm{C})$, conductance $(\mathrm{G})$ and leakage current density of the device and to supply the required voltage bias across the MOS capacitor. The LCR meter and $\mathrm{pA}$ meter is controlled by a personal computer using the LabVIEW program described earlier. The PC is connected to the LCR meter and pA meter via GPIB interface.

Figure 4 shows the connection diagram using four probes to the device under test. For accurate measurements, the lid of the box must be covered at all times. This is because when a semiconductor is exposed to light, electrons can get excited from the valence band into the conduction band by the absorption of photons, provided the photon energy is greater than $\mathrm{E}_{\mathrm{g}}$. The LCR meter and $\mathrm{pA}$ meter has the two high voltage $(\mathrm{H})$ terminals connected to the high probe of the device under test (DUT) box and the two low voltage (L) terminals connected to the low probe of the DUT box.

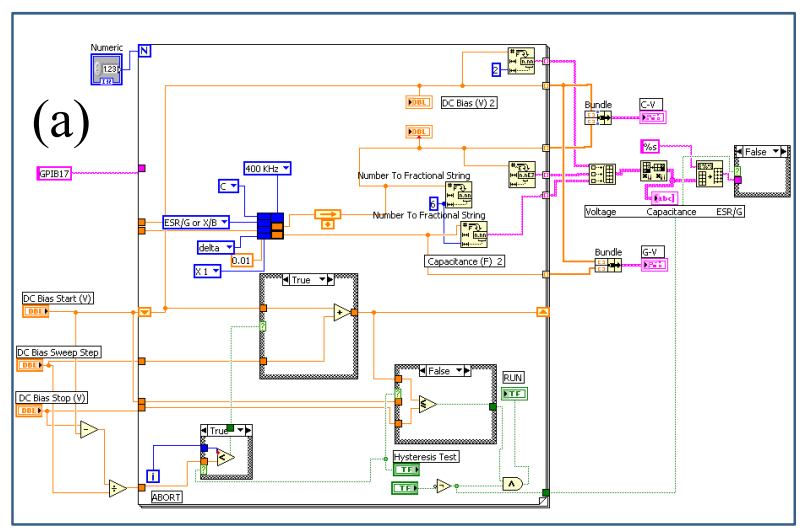

Figure 2(a): LabVIEW function diagram for 4275A LCR meter.

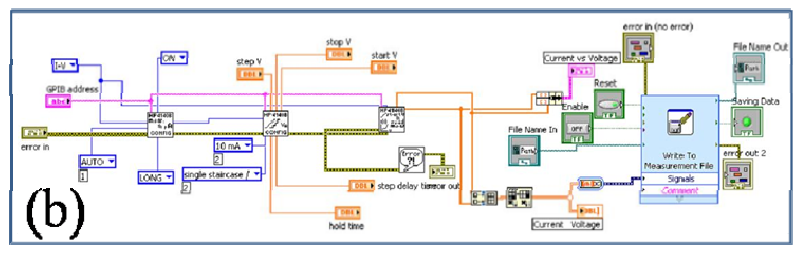

Figure 2(b): LabVIEW function diagram for 4140B pA meter. 


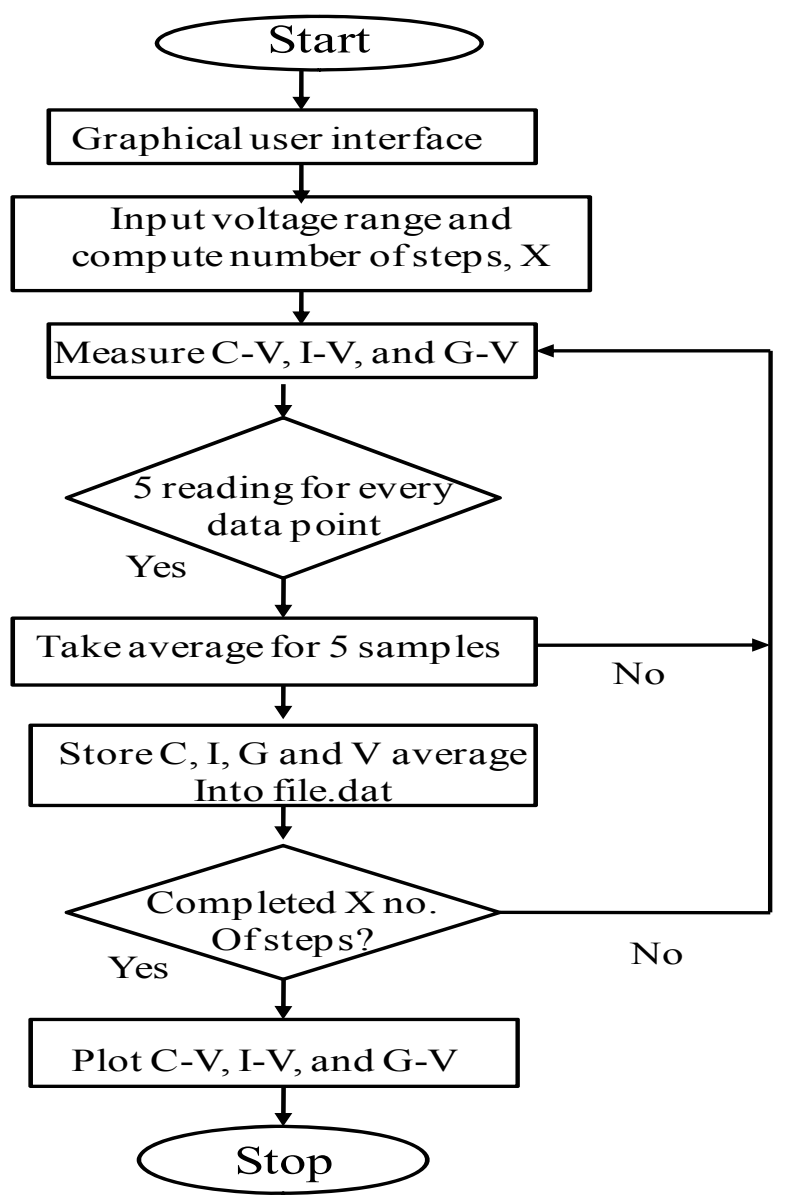

Figure 3: Flow chart showing operation of the LabVIEW Program.

As shown in Figure 4, the two $\mathrm{H}$ terminals are connected to the high terminals of DUT via coaxial cables. This is also same for the two L terminals. The control of instruments has been realized by the LabVIEW software tool as described earlier.

$\mathrm{C}-\mathrm{V} / \mathrm{G}-\mathrm{V}$ characteristics are defined as small-signal capacitances/conductance and are measured by applying a small ac voltage on top of a de bias across the MOS capacitor and sensing the out-of-phase ac current at the same frequency. In this study, the $\mathrm{C}-\mathrm{V}$ and $\mathrm{G}-\mathrm{V}$ characteristics measurements were performed at various frequencies $(10$ $\mathrm{kHz}-10 \mathrm{MHz}$ ) by precision LCR meter. J-V characteristics are measured using semiconductor parameter analyzer. Measured capacitance-voltage, conductance-voltage and leakage current density vs. voltage of MOS capacitor are shown in Figures 5(a), (b), and (c), respectively.

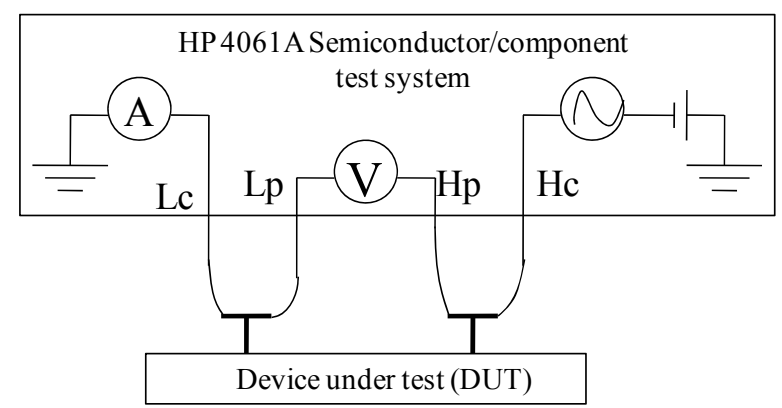

Figure 4: HP4275A LCR meter and HP4140B pA meter connection to the MOS capacitor.

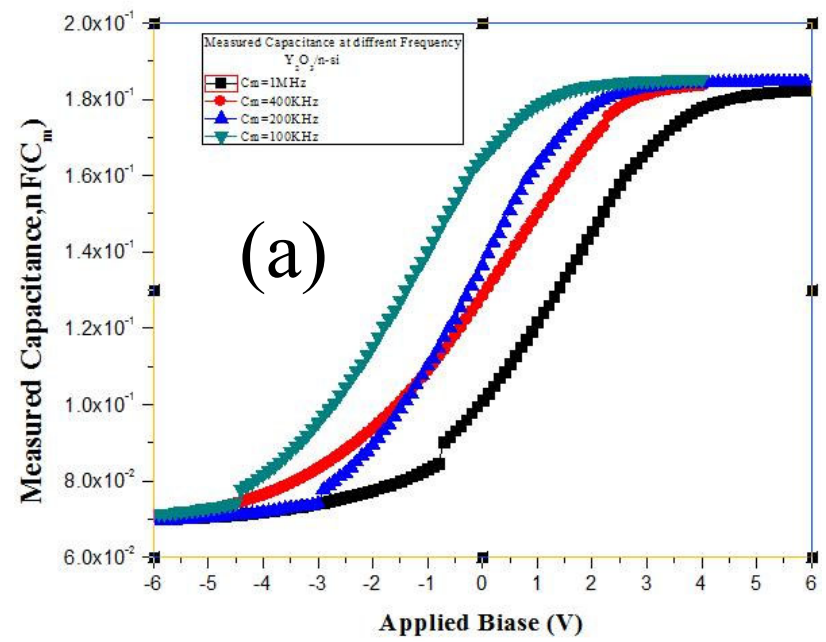

Figure 5(a): Measured C-V characteristics at room temperature at different frequencies.

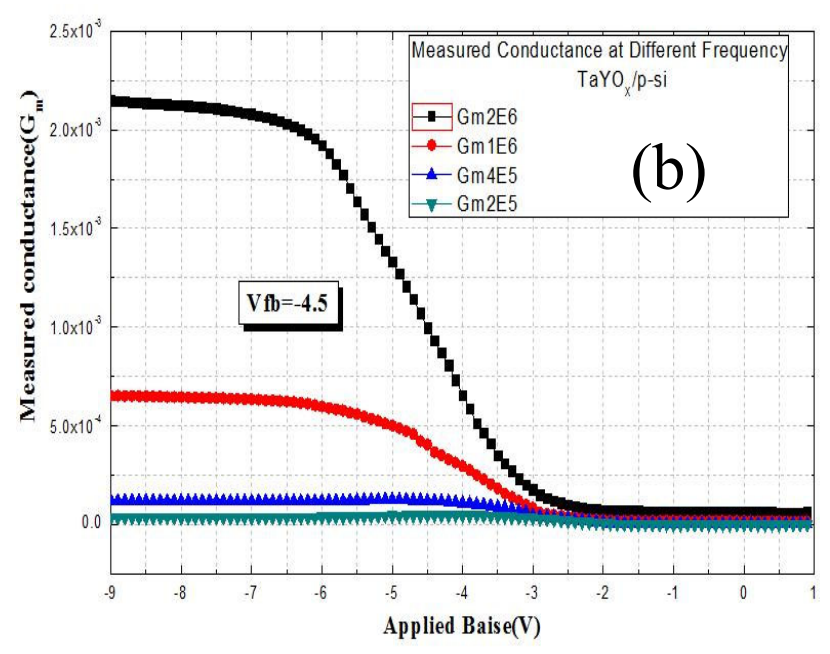

Figure 5(b): Measured G-V characteristics at room temperature at various frequencies.

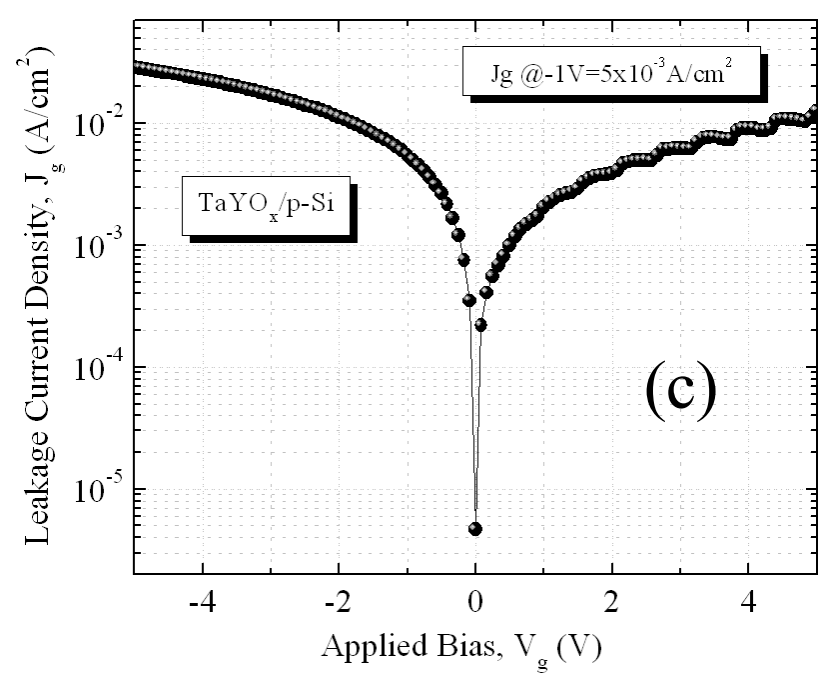

Figure 5(c): Measured current-voltage (I-V) characteristics at room temperature. 


\section{ONLINE IMPLEMENTATION}

To view a LabVIEW front panel remotely using a Web browser, the server has to be configured first. The following steps are used to configure the server so a client computer can view an application front panel remotely using a browser. The following steps were followed to put the developed tool on the Internet.

Enable the Web Server on the server computer.

Configure the browser access list so the client computer can connect to the server.

The application is running or the VIs whose front panels - clients want to view - are in memory on the server computer.

Create an HTML file using the Web Publishing Tool. An existing HTML file can also be used. The WIDTH and HEIGHT parameters of the $<$ OBJECT $>$ and $<$ EMBED $>$ tags can also be changed to customize the application or front panel appearance in the client browser.

VIs that will be viewed or controlled remotely, should be as small front panels as possible since Web Server uses more system resources to load and display large front panel images than small front panel images, which can slow the execution of the VI itself.

For a client computer to view a front panel remotely using a browser:

Install the LabVIEW Run-Time Engine.

Launch the web browser.

In the URL of the browser, type the IP address or computer name of the server followed by the name of the HTML file. When a front panel is viewed remotely from a browser, the application or VI you want to view must be running on the server computer.

\section{CONCLUSION}

A program based on LabVIEW was developed to characterize $\mathrm{Y}_{2} \mathrm{O}_{3}$ and $\mathrm{Ta}_{2} \mathrm{O}_{5}$ high-k gate dielectrics using
HP4275A LCR meter and HP4140B pA. The automated measurement setup has been deployed via internet for online MOS capacitor characterization and parameter extraction in real time. On the front end, the user interacts with the system through a graphical user interface implemented through Internet web browser. On the back end, the Server processes experiment requests from users and runs them. Once the experiment is completed, the Server sends the measured data to the Client for display on the screen and further manipulation by the user. Furthermore, the design takes the advantage web browser framework for provision of authentication and authorization services, as well as common administrative tasks, such as, user management and logon/logoff assignments.

\section{REFERENCES}

[1] LabVIEW 8.5 User manual, Measurement and automation, National Instruments Inc. (Aug. 2007).

\section{AUTHORS}

S. C. Pandey is an M. Tech student in the specialization of Microelectronics and VLSI Design at IIT Kharagpur (e-mail: subhashiitkh@gmail.com).

A. Maiti is an undergraduate student studying computer applications in Vidyasagar University, Midnapur, India (e-mail: anandamaiti@live.com).

T. K. Maiti is a PhD student and is with the Electronics and Electrical Communication Engineering Department, IIT Kharagpur (e-mail: tkm.iitkgp@yahoo.com).

C. K. Maiti is the Head of and Professor at the Electronics and Electrical Communication Engineering Department, IIT Kharagpur, (Phone no: +91-(0)3222281475, e-mail: ckm@ece.iitkgp.ernet.in)

This work was supported in part by the MHRD, Govt. of India. This article was modified from a presentation at the REV2009 conference in Bridgeport, CT, USA June 2009. Submitted, March 5,2009. Published as resubmitted by the author(s) on July, 20, 2009. 